\title{
Relação das obras entradas na Biblioteca da Faculdade de Direito de São Paulo, no período compreendido entre 15 de Outubro de 1933 e 15 de Fevereiro de 1934
}

OBRAS GERAIS (0)

Dicionarios, Enciclopédias, Revistas, Estatutos, etc

Afonso Duarte Ribeiro-Anuario de legislação de Fazenda-Rio de Janeiro, 1917, 1919, 1920 e 19.28-5 vols. -Compra.

Anales de la Universidad de Chile1932-1933-3 vols.-Permuta.

Anales de la Universidad de ChileSuplemento-1932-1 vol.-Permuta.

Annales de l'Université de ParisSorbonne, 1933-1 vol:-Permuta.

Annals (The-)-Philadelphia, 19331934-2 vols.-Compra.

Anuario da Escola Politecnica de São Paulo-1933-1 vol.-Doação.

Anuario da Escola de Sociologia e Politica de São Paulo-S. Paulo, 1933$1934-1$ vol.-Doação.

Annuario della $R$. Universitá di Torino (1932-1933)-Torino, 1933-1 vol.Permuta.

Archivos de la Universidad de Buenos Aires-1932-B. Aires, 1933-1 vol.Permuta.

Arquivos do Instituto Biologico de Defesa Agricola e Animal-S. Paulo, 1928-1931-2 vols.-Doação.

Arquivos do Museu Narional-Vol. XXX
—Rio de Janeiro, 1928-1 vol.-Doação.

Boletim de Agricultura-S. Paulo, 1932 -1 vol.-Doação.

Boletim de Educação Publica-Rio de Janeir, 1930-1932-4 vols.-Doação.

Boletim do Laboratorio de Ensaio de Materiais da Escola Politecnica de São Paulo-S. Paulo, 1931-1933-3 vols.-Doação.

Baletim da União Pan-AmericanaWashington, 1933-1 vol.-Permuta.

Boletin Mensual del Seminario de Ciencias Juridicas y Sociales-B. Aires, 1932,-1 vol.-Permuta.

Bulletin of Wassar College-N. York, 1934-1 vol.-Doação.

Chavigny (P.-)-Organisation du travail intellectuel-Paris, $1920-1$ vol. -Doação.

Comentario (O-) -Revista quinzenal -S. Paulo, 1931-1 vol-Doação.

Crozet (Leó-) $\rightarrow$ Manuel pratique du bibliothécaire-Paris, $1932-1$ vol.Doação.

D. N. C.-Revista do Departamento Nacional do Café-Rio de Janeiro, 1933 -1 vol.-Doação.

Dalloz-Dictionnaire pratique de droit -Paris, 1 vol.-Compra.

Direito e Letras-Revista academicaS. Paulo, 1878-1 fasc.-Daação.

École Libre des Sciences Poditiques- 
Organisation et programme des cours-Paris, 1933-1 vol.-Doação.

Egatéa-Revista da Universidade Tecnica do Rio Grande do Sul-PortoAlegre, 1931-1932-1933-3 vols_-Doação.

Esprit International (L'-)-Paris, 1933 -1 vol.-Doação.

Estudos de Criminologia e de Psicanalise -Rio de Janeiro, 1932-1 vol. -Doação.

Estudos Juridicos e Sociais-Rio de Janeiro, 1933-1 fasc.-Doação.

Europe-Revista trimestral- $\longrightarrow$ Paris, 1933 -3 vols.-Compra.

Encyclopaedia of the Social SciencesN. York, 1933-3 vols.-Compra.

Feira Literaria-S. Paulo, 1927 e 1928 -3 vols.-Doação.

Gazeta Juridica-São' Paulo, 1893-1914 -62 vols. - Doação.

Idort-Orgão do Instituto de Organização Racional do Trabalho-S. Paulo, 1933-2 vols.-Doação.

International Conciliation-N. York, 1933-1 vol.-Doação.

J. Guimarães Menegale-O que é e o que deve ser a bibliotéca publicaB. Horizonte, 1932-1 vol.-Doação.

Journal des Économistes-Paris, 19321 vol.-Cotmpra.

Jurisprudencia-Pub. do "Diario da Justiça"-Rio de Janeiro, 1933-2 vols.-Compra.

Justiça-Revista mensal-Porto Alegre, 1933-1 vol.-Permuta.

Livret de l'Étudiant-Université de Paris, 1933-1934-1 vol. - Doação.

Memoria-Anuario-Curso Academico de 1929-1930-Universidad de Habana1 vol.-Permuta.

Mensagens Apresentadas ao Congresso Legislativo, Pelo Dr. Altlno Arantes, Presidente do Estado de São PauloS. Paulo, 1916-1919-4 vols.-Doação.

Mois (Le-)-Paris, $1933-4$ vols.Doação.

Onze de Agosto (O-)-S. ¡Paulo, 1933 -1 vol.-Doação.

Ordem (A-)-Rio de aneiro, 1930 a 1933-4 vols. - Doação.

Patentes e Marcas-Revista mensal de propriedade industrial-S. Paulo, 1933 -1 vol.-Doação.
Paraná Judiciario-Curitiba, 1933-1 vol.-Permuta.

Programa e Regulamento da Primeira Conferencia de Estatistica a Realizar-se de 12 a 20 de Outubro de 1930 -Rio de Janeiro, 1930-1 vol._Doação.

Relatorio de 1918 Apresentado á Camara Municipal de S. Paulo pelo Prefeito Washington Luis Pereira de Souza-1 vol.-S. (Paulo, 1919-Doação.

Rentrée Solennelle des Facultés-Université de Montpellier-Montepellier, 1933-1 voll.--Doação.

Repertorio de Direito Comercial Brasileiro-S. Paulo, 1931-2 fasc.-Doação.

Revista do Arquivo Publico Mineiro$1900,1907,1912,1927,1928$ e 1929 B. Horizonte-6 vols.-Doação.

Revista de Critica Judiciaria-Rio de Janeiro, 1933-1 vol.-Compra.

Revista do Brasil-S. Paulo, 1916-1923 -11 fasc.-Permuta.

Revista Cubana de Derecho-Habana, 1932-1 vol.-Permuta.

Revista de Derecho y Legislación-Caracas, 1918-1919- -2 vols —Permuta.

Revista de Direito Civil, Comercial e Criminal (Bento de Faria)-Rio de Janeiro, 1933-1 vol.-Compra.

Revista de Educação-S. Paulo, 19311933-16 vols.-Doação.

Revista de Estadistica Municipal de la Ciudad de Buenos Aires-B. Aires, 1931-1932-2 vols.-Doação.

Revista dla Faculdade de Direito da Baía-Baia, 1933-1 vol.-Permuta.

Revista da Faculdade de Direito de São Paulo-1893-1898 e 1903-7 vols.iP'ermuta.

Revista de Identificacion y Ciencias Penales-1931-1932-1 vol.-Permuta.

Revista do Instituto de Café do Estado de São Paulo-S. Paulo, 1932-1 vol. -Doação.

Revista do Instituto Histórico e Geografico Brasileiro-Rio de Janeiro, 1927-1929-3 vols.-Doação.

Revista Juridica-Pernambuco-Recife, 1932 —2 vols.-Piermuta.

Revista de Jurisprudencia-Rio de Janeiro, 1933-1 vol--Permuta. 
Revista de Portugal (Eça de Queiroz) -Porto, 1889_4 vols.-Permuta.

Revista dos Tribunais-S. Paulo, 19332 vols.-Doação.

Revista da Universidade do Rio de Janeiro-Rio de Janeiro, 1932 e 19333 vols.-Permuta.

Revista Universitaria-Organo de la Universidad Menor del Cuzco-Perú, 1932-1933-2 volr.-Permuta.

Rivista del Diritto Commerciale-Milano, 1923-1928-12 vols-Compra.

Rivista Internazionale di Filosofia del Diritto-Roma, 1933-1 vol.-Compra.

Revue de Droit International PrivéParis, 1933-1 vol.-Compra.

Revue Philosophique de la France et die l'Etranger-Paris, 1933-1 vol.Compra.

Radrigo Otavio-Dicionário de Direito Internacional ¡Privado-Rio de Janeiro: $1933-1$ vol.-Doação.

Saint-Adolphe, J. C. R. Milliet de-Dicionario geografico, historico e descritivo do Imperio do Brasil-Paris, 1863-2 vols.-Permuta.

São Paulo Judiciario-S. Paulo, 19031914-36 vols.-Doação.

Vie Intellectuelle (La-)-Paris, 19332 vols.-Doação.

\section{FILOSOFIA (1)}

\section{Filosofia, Psicologia, Espiritismo,} Moral, etc.

A. Pompêo-Deus e Patria-S. Paulo, 1934-1 vol.-Doação.

Corinto Barbosa-Doutrina evolucionaria-Rio de Janeiro, 1933-1 vol.Doação.

D'Agen, Boyer - Ave Caesar!-Paris, 19.25-1 vol.-Doação.

Dubois, 'Paul-Raison et sentimentBerne, 1911-1 vol.-Doação.

Eurico de Goes-A corrente filosofica do seculo-Rio de Janeiro, 1912-1 vol.-Doaçãı.

Fabre-Luce, Alfred-Pour une politique sexuelle-Paris, 1933-1 vol.-Doação.

Franco da Rocha-O Pansexualismo na doutrina de Freud-S. Paulo, 19201 vol.-Doação.

Ingenieros, José-El hombre mediocre -B. Aires, 1918-1 vol.-Doação.
Knudsen, K. A. Wieth-Le conflit des sexes dans l'évolution sociale et la question sexuelle-Paris, 1931-1 vol. -Compra.

Kolontai, Alexandra-A nova mulher e a moral sexual-S. Paulo, 1932-1 vol.-Doação.

Le Bon, Gustave-La psychologie politique-Paris, 1919-1 vol.-Doação.

Lemos Britto-Psicologia do adulterio -Rio de Janeiro, 1933-1 vol.-Compra.

Nicolle, Charles-Biologie de l'invention—Paris, 1932-1 vol.-Doação.

Pilo, Mario-Estetica-Lezioni sul gusto-Milano, 1906-1 vol.-Doação.

Pontes de Miranda-A sabedoria dos instintos-Rio de Janeiro, 1921-1 vol.-Doação.

Richet, Charles-Notre sixième sensParis, s/d-1 vol.-Doação.

Yasoroku, Soyeshima-The essence of Bushido-Tokyo, s/d-1 vol.-Doação.

Weis, P. et Foix, G.-Le magnétismeParis, 1926-1 vol.-Doação.

Zweig, Stefan-Nictzche-Rio de Janeiro, 1934-1 rol.-Permuta.

\section{RELIGIŐES (2)}

Abdrusckin-Na luz da verdade, São Paulo, 1933, 2 vols.-Doação.

Baudot, Jules-Le Bréviaire romain, Paris, 1908, 1 vol.--Doação.

Baudot, Jules-Notions générales de liturgie, Paris 1908, 1 vol.-Doação.

Benarus, Adolfo-Os judeus, Lisboa, s/d., 1 vol.-Doação.

Boeglin, Eugène-Le crépuscule du luthérianisme, Paris, 1907, 1 volDoação.

Bourlon, I.-Les assemblées du clergé et le protestantisme-Paris, 1909, 1 vol.-Doação.

Brémond, Henri-L'évolution du clergé anglioan-Paris, s/d., 1 volDoação.

Brémond, Henri-La littérature religieuse d'avant-hier et d'aujound'hui. Paris, 1908, 1 vol.-Doação.

Cauzons, Th. de-Les vaudois et l'Inquisition-Paris, 1908, 1 vol.-Doação.

Cauzons, Th. de-Les albigeois et l'Inquisition-Paris, 1908, 1 vol.-Doaçāo. 
Dottin, Georges-Les livres de Saint Patrice-Paris, 1909, 1 vol.-Doação.

Ermani, V.-Saint Paul et la prièreParis, 19017, 1 vol.-Doação.

Gastonné, Amédée-L'elau bénite-Paris, 1907, 1 vol.-Doação.

Graziani, Paul-Boniface VIII, Paris, s/d.-Doação.

João do Rio-As religiões no Rio-Rio de Janleiro, 's/d., 1 vol.-Doação.

Manuel E. Altenfelder Silva-Brasileiros heróis da fé-São Paulo, 1928, 1 vol.-Doação.

Maritain, Jacques-Religion et culture -Paris, 1922, 1 vol.-Doação.

Piolet et Vadot-L'Eglise Catholique dans le Continent Noir $\longrightarrow$ Paris, 1908, 1 vol.-Doação.

Piolet et Vadot-L'Eglise Catholique aux Indes-Paris, 1907, 1 vol.-Doação.

Porque ser Antisemita? Inquerito entre os intelectuais brasileiros-Rio de Janeiro, 1933, 1 vol.-Douação.

Protocole dies Sages ide Sion-Paris, 1931, 1 voll.-Doação.

Roberto Seidl-Nisia Floresta, Rio de Janeiro, 1933, 1 vol.-Doação.

Rouquette, J־Les victimes de Chalvin -Paris s/d-1 vol.-Doação.

Ronquette, J.-Les Saint-Barthélemy calvinistes-Paris, 1908, 1 vol.-Doação.

S. Vieira Souto-Comte santificado por Clotilde-Rio de Janeiro, 1929, 1 vol. -Doação.

Saint-Jerôme-Vie de Paul de Thèbes et vie h'Hilarion-Paris, 1908, 1 vol. -Doação.

\section{CIENCLAS JURIDICAS E SOCLAIS (3)}

Sociologia, Politica, Estatistica, Economia, Direito, Medicina Legal, Educação, Costumes, etc.

A. Bento de Faria-Pareceres, vol. 1Rio de Janeiro, 1933-Compra.

A. Sampaio Doria-O comunismo caminha no Brasil-São Paulo, 1933, 1 vol.-Doação.

Aben-Attar Neto-Integralismo-Rio de Janeiro, 1934, 1 vol.-Doação.

Abner de Moura-Os centros de inte- rêsse na Escola-Sãio Paulo, 1931, 1 vol.-Doação.

Achilles Bevilaqua-Codigo Comercial Brasileiro-Rio de Janeiro, 1932-1 vol.-Compra.

Adalberto Garcia-No plenario-São Paulo, 1913, 1 vol.-Dolação.

Afonso de Toledo Bandeira de MelloPolitica comercial do Brasil, Rio de Janeiro, 1933, 1 vol.-Doação.

Aires da Matta Machado Filho-Educação dos cegos no Brasil, Belo Horizonte, 1931, 1 vol.-Doação.

Alain-Le citoyen contre les pouvoirs -Paris, 1926, 1 vol.-Doasão.

Albertino Moreira-Terra de ninguem -São Paulo, 1932, 1 vol.-Doação.

Alceu Amoroso Lima-Introdução á Economia moderna.-Rio de Janeiro, 1933, 1 vol.-Compra.

Alfredo João Louzada-Legislação social-trabalhista.-Rio de Janeiro, 1933, 1 vol.-Compra.

Almaquio Diniz-Do depósito. Não pode ser idepositário o proprietário da cousa-Rio de Janeiro, 1930, 1 vol.Compra.

Antonio Luiz da Camara Leal-Comentários ao Código de processo civil e comercial do Estado de São PauloSão Paulo, 1930-1933-Doação.

Antonio Manuel Fernandes-Indice cronologico, explicativo e remissivo da legislação brasileira desde 1822 até 1848_Rio de Janeiro, 1872, 3 vols.Doação.

Antonio Sieveriano de Andrade e Silva -Medicina legal aplicada-Rio de Janeiro, 1924, 1 vol.--Doação.

Arlindo Veiga dos Santos-Para a ordiem nova-São Paulo, 1933-1 vol.Doação.

Artur Lara Ulrich-Defesa do Cioronel João Francisco Pereira de Souza Sant'Anna do Livramento, 1903, 1 vol. -Doação.

Assessor Fiscal do Estado de São Paulo -São Paulo, 1933, 1 vol-Compra.

Asúa, L. Jimenez de-Código penal de la Rusia Soviética-Madrid, 1927, 1 vol.-Compra.

Asúa, L. Jimenez de-Crónica idel crimen--Madrid, 1929, 1 vol.-Compra.

Asúa, L. Jimenez de-La pericolositáTorino, 1923, 1 vol.-Compra. 
Asúa, L. Jimenez de-Il delitto de contagio venered-Torino, 1929, 1 vol.Compra.

Augusto Cesar-Pela nova Constituição Politica do Brasil-Rio de Janeiro 1931, 1 vol.-Doação.

Augusto Cesar-A verdadeira questão social-Rio de Janeiro, 1931, 1 vol.Doação.

Augusto Ferreira de Castilho-Democracia no Brasil--São Paulo, 1929, 1 vol.-Doação.

Bella, Vincenzo de-Il reato di assoziazione a delinquere-Torino, 1933, 1 vol. $\longrightarrow$ Compra.

Bento Carqueja-Economia PoliticaTomo 1-Porto, 1926, 1 vol.-Doação.

Berdiaeff, Nicolas-Le christianisme et la Iutte des classes-Paris, 1933, 1 vol.-Doação.

Bernardino de Lima-Direito e economia politica-São Paulo, 1882, 1 vol. -Doação.

Bierta Lutz-A nacionalidadle da mulher casada-Rio de Janeiro, 1933, 1 vol. -Doação.

Boaventura Nogueira da Silva-O envenenamento em face da lei penalSão Paulo, 1927-1 vol.-Doação.

Borges de Medeiros- 0 poder moderador na República presidencial-Pernambuco, 1933, 1 vol.-Compra.

Bourbousson, E-Traité général de la nationalité dans les cinq parties du monde-Paris, 1931, 1 vol-Permuta.

Braz di Francesco-Pela cultura policial-São Paulo, 1931, 1 vol_-Doação.

Brogan, D. W.-The american political system-Londion, 1933-1 vol.-Compra.

Calón, E. Cuello-El derecho penal ruso -Barcelona, 1931, 1 vol.-Compra.

Calón, E. Cuello-Exposición del códígo penal reformado de 1932-Barcelona, 1933, 1 vol.-Compra.

Candido Motta Filho-Alberto Torres e o tema da nossa geração-Rio de Janeiro, 1931, 1 vol.-Doação.

Candido de Oliveira Filho Pratica civil, vol. $\mathrm{X} \longrightarrow$ Rio de Janeiro, 1934, 1 vol.-Doação.

Carlos Sampaio-Do divórcio-São Paulo, 1911, 1 vol.-Doaçâo.

Carlos Sampaio-Do casamento-São ¿Paulo, 1923, 1 vol.-Doagão.
Carneiro Maia-Código dos Municípios do Estado de São Paulo, São Paulo, 1929, 1 vol-Doação.

Carnelutti, Francesco-Studi di diritto processuale-Padova, 1925, 1 vol.Compra.

Cervantes, Manoel-Historia y naturaleza de la personalidad juridicaMexico, 1932, 1 vol.-Doação.

Cesar Tripoli-Elementos de direito civil e comercial-São Paulo, 1932-2 vols.-Doação.

A. C. de Salles Junior-Reforma de tarifas-S. Paulo, 1932-1 vol.-Doação.

Chimienti, P-Droit Constitutionnel Italien-Paris, 1932-1 vol-Compra.

Cristofaro, Carlo-Trattato del Diritto d'Autore e d'Inventore-Torino, 1931 -1 vol.-Compra.

Cicero Ferreira Lopes-Peculato, moeda falsa e falsificação de documentos-Rio de Janeiro, 1934-1 vol.Compra.

Coleção das Leis e Decretos do Estado de São Paulo-1906-1914 e 1933-4 vols.-Compra.

Coleção de Leis e Decretos do Estado de Sergipe-Aracajú, 1903-1928-11 vols.-Doação.

Coleção de Leis, Decretos e Regulamentos do Estado doi AmazonasManaus, 1902-1929-26 vols.-Doação.

Coleção das Leis da Republica dos Estados Unidos do Brasil-Rio die Janeiro, 1932-5 vols.-Compra.

Constituição do Estado Ide Sergipe do Ano de 1901-Aracajú, 1903-1 vol.Doação.

Constituição Politica de la Republica del Chile-Santiago, 1931-1 vol.Doação.

Cortesani, Giuseppe-La responsabilitá nel diritto aereo-Tơrino, 1929-1 vol-Compra.

Crothers, George Edward-The educational ideals of Jane Lathrop Stanford-California, 1933-1 vol.-Doação.

Davis, Horace-The Judicial Veto-Boston-N. York, 1914-1 vol.--Doação.

Decretos e Atos da Intervenção Federal no Amazonas-Manaus, 1925-1 vol.-Doação.

Delgado de Carvalho-Sociologia edu- 
cacional-S. Paulo, 1933-1 vol.-Doação.

Deploige, Simon-Le conflit de la morale et de la sociologie-Paris, 1933 1 vol.-Compra.

Dona, Gaetano- 11 silenzo nella teoria delle prove giudiziali-Torino, 1929 -1 vol.-Compra.

Ernani Lins da Cunha-Codigo do Processo Civil e Comercial do Estado de S. Paulo-S. Paulo, 1932-1 vol.Doação.

Ernesto Luiz d'Oliveira-Replica á conferencia "Do Microbio ao Homem" -S. Paulo, 1928-1 vol.-Dpação.

Esquilo de Oliveira-Embargos n. 18.822 -Assis-S. Paulo, 1933-1 vol.-Doação.

Estatistica Agricola Zootecnica-19311932-S. Paulo, 1933-1 vol.-Doação.

Estatistica Intelectual do Brasil-1929 -Rio de Janeiro, 1932-1 vol.-Doação.

Estatistica das Instituições de Assistencia a Enfermos com Internamiento em 1930-Rio de Janeiro, 19331 vol.-Doação.

Estevam A. de Oliveira e Teodomiro Dias-Tutela-S. Paulo, 1933-1 vol.Doação.

Evaristo de Morais-A escravidão africana no Brasil-S. Paulo, 1933-1 vol.-Compra.

F. Nicolau Ançarah-Executivo hipotecario-Razões-S. Paulo, 1933-1 vol. —Doação.

Fanciulli, Giuseppe-L'individuo nei suoi rapporti sociale-Torino, 19051 vol.-Permuta.

Feder, Gottifried-Der Deutsche Staat auf nationaler und socialer Brundlage-Munchen, 1933-1 vol.-Doação.

Fernando Andrade Peres de Lima-O casamento putativo no Direito Civil Português-Coimbra, $1930-1$ vol.Compra.

Fiorentino, Adriano R.-Emigrazione transoceanica-Roma, 1931-1 vol.Compra.

Firmino Whitaker-Condenação condicional-S. Paulo, 1930-1 vol.-Daação.

Firmino Whitaker-Desapropriação-S. Paulo, 1927-1 vol.-Doação.
Francisco Brant-Das aposentadoriasB. Horizonte, 1913-1 vol.-Doação.

Francisco Brant--O Congresso Postal Sul Americano-B. Horizonte, s/d.1 vol.-Doação.

Francisco Brant-Escritos de direitoB. Horizonte, s/d.-1 vol.-Doração.

Francisco José de Almeida Brant-Comentarios ao regulamento dos Correios do Brasil-B. Horizonte, 19111 vol.-Doação.

Gatti, Tancredi-L'escezione penalePadova, 1933-1 vol.-Compra.

Getzeny, H.-Capitalismo e socialismo -P. Alegre, 1933-1 vol.-Doação.

Gilberto Sampaio-Falencias-S. Paulo, s/d.-1 vol.-Doação.

Gilberto Sampaio-Debaties forensesS. Paulo, s/d.-1 vol.-Doação.

Gilberto Sampaio-Reserva de dominio-Reclamação neivindicatoria $-S$. Paulo, 1928-1 vol.-Doação.

Girola, Carlo-Teoria dell decentramento amministrativo-Torino, 1929 -1 vol.-Compra.

Godofredo de Faria-O mal é o protecionismo-Exame da situação economica do Brasil-Rio ide Janeiro, 1932-1 vol.-Doação.

Gustavo Lessa-O govêrno e a educação-Rio de Janeiro, 1932-1 vol.D’oação.

Haroldo Valladão-Da cooperação internacional nos processos criminaisS. Paulo, 1933-1 vol.-Doação.

Henrique Coelho- $O$ poder legislativo e o poder executivo no Direito $\mathrm{Pu}$ blic'o Brasileiro-S. Paulo, 1905-1 vol.-Doação.

Historia Universal del ProletariadoBarcelona, s/d.—2 vols.-Doação.

Hipolito de Camargo-Jurisprudencia referente ao Codigo Penal-S. Paulo, 1901-1 vol.-Doação.

Ichausti, Amado-Origines del poder economico de la iglesia-Madrid, 1932 -1 vol.-Doação.

Instruções aos Delegados Gerais e Seccionais e ás Comissões CensitariasRio de Janeiro, 1920-1 vol.-Doação.

J. C. Ataliba Nogueira-As loterias iestaduais e o fisco-S. Paulo, 1930-1 vol.-Doação.

J. C. Ataliba Nogueira-A reforma da 
Constituição Federal-S. Paulo, 1926 -1 vol.-Doação.

J. J. Cardozo de Mello Junior e J. J. Cardozo de Mello Neto-Apelação civil n. ${ }^{\circ} 18.537-$ Capital-S. Paulo, 1925 -1 vol.-Doação.

J. J. Cardozo de Mello Junior e J. J. Cardozo de Mello Neto.-Apelação civil n: ${ }^{\circ} 14.155$-Capital-S. Paulo, 1925 -1 fol.-Doação.

J. J. Cardozo de Mello Junior e J. J. Cardozo de Mello Neto.-Apelação civil n. ${ }^{\circ}$ 20.453-Capital-S. Paulo, 1933 -1 vol.-Doação.

J. J. Cardozo de Mello Neto-O fascismo e a economia politica-S. Paulo, 1931-1 vol.-Doação.

J. Lafayette-Exercito de opereta-A fedieralização das policias estaduais -S. Paulo, 1933-1 vol.—Doação.

J. M. Azevedo Marques-A HipotecaS. Paulo, 1933-3.a ed.-1 vol.-Doação.

J. M. de Carvalho Santos-Codigo Civil Brasileiro interpretado-1.' vol.-Rio de Janeiro, 1934-1 vol--Compra.

J. Pandiá Calogeras-Problemas de administração-S. Paulo, 1933-1 vol.Compra.

J. Peçanha de Figueiredo-Comércio de Banco-S. Paulo, 1931-1 vol-Doação.

J. Pinto Antunes-A filosofia do Estado moderno-S. Paulo, 1934-1 vol. -Doação.

J. Rodrigues de Mereje-Sociologia geral-S. Plaulo, 1933-1 vol.-Doação.

J. X. Carvalho de Mendonça-Pareceres-Rio de Janeiro, 1933-1 vol_Compra.

João Arruda e Plinio Barreto-Questões de dominio-S. Paulo, 1927-1 volDoação.

João Evangelista Rodrigues-Crodigo do Pracesso Civil e Comeraial do Estado de S. Paulo--S. Paulo, 19301 vol.-Doação.

João Francisco-Carta ao governo revolucionario-Rio de Janeiro, 1934 -1 vol.-Doação.

João Francisco da Cruz-Tratado de policia-S. Paulo, 1932-1 vol.-Doação.

João Mendes de Almeida Junior-Os indigenas do Brasil, seus direitos individuais e politicos-S. Paulo 1912-1 vol.-Doação.

João Neves da Fontoura e Cardozo de Mello Neto-Embargos civis do Estado do Paraná-S. Paulo, 1932-1 vol.-Doação.

José Afonso Mendonça de AzevedoElaborando a Constituição Nacional -B. Horizonte, 1933-1 vol-Compra.

José 'Alberto dos Reis-Breve estudo sôbre a reforma do processo civil e comercial—Coimbra, $1933-4$ vol:Compra.

José Bonifacio-Reimpressão de um opusculo raro de José Bonifacio sôbre a emancipação ıdos escravos no Brasil-Rio de Janeiro, 1884-Doacão.

José Bonifacio de Andrada e SilvaRepresentação á assembléa geral constituinte e legislativa do Brasil sobre a escravatura-Paris, 18251 vol.-Doação.

José Julio Soares-Sociedades cooperativas-Rio de Janeiro, 1933-1 vol., -Compra.

José Ovidio Marconides Romeiro-Sentenças e decisões-Rio de Janeiro, 1926-1 vol.-Doação.

Julgados e decisões do superior Tribunal de Justiça do Estado do Amazonas-Manaus, 1933-1 vol.-Doação.

Juvenal Lamartine-O meu govêrnoRio de Janeiro, 1933-1 vol.-Doação.

Kokovtzoff, W.-Le bolchévisme à l'oeuvre-Paris, 1926-1 vol.-Doação.

Lacerda de Almeida-Dos efeitos das obrigações-Rio de Janeiro, 19341 vol.-Compra.

Legislação Cafeeira do Brasil-Rio de Janeiro, 1933-1 vol.-Doação.

lehmann, Otto-La internacional sangrenta de los armamentos-Madrid, 1929-1 vol.-Doação.

Leis e decretos do Estado do PiauiTerezina, 1910, 1923 a 1928, 1931 a 1933 -10. vols.-Doação.

Leis do Estado do Paraná-Curitiba, 1924-1926-3 vols.-Doação.

Leone, E-Teoria della politica-Torino, 1931-1 vol--Compra.

Lessona, Silvio-Trattato di diritto sanitario-Torino, $1914-1921-2$ vols. -Compra. 
Leven Vampré-São Paulo, terra conquistada..-S. Paulo, 1932-1 vol.Doação.

Luiz Candido de Almeida Leite-Lei das falencias-Dec. n. 5746, de 9-121929-S. Paulo, 1930-3.a. ed.-1 vol. -Doação.

Luiz Pereira de Campos VergueiroCarateristico do estado de falencias -S. Paulo, 1904-1 vol.-Doação.

M. Bomfim-O Brasil na AmericaRio de Janeiro, 1929-1 vol-Doação.

M. I. Carvalho de Mendonça-O poder judiciario no Brasil-Curitiba, 18991 vol.-Doação.

M. I. Carvalho de Mendonga-A intervenção e 'a doutrina de MonroeCuritiba, 1896-1 vol.-Doação.

Manoel Carlos de Figueiredo FerrazDecisões-S. Paulo, 1931-1 vol.Doação.

Manuel Olimpio Romeiro-S. Paulo e Minas na economia nacional-S. Paulo, 1930 -1 vol.-Doação.

Mario de 'Assis Moura-Instituto das correições no Estado de S. PauloS. Paulo, 1931-1 vol.-Doação.

Mario de Assis Moura-Pratica das demarcações e divisões regundo o Coıdigo do Processo Sivil e Comercial do Estado de S. Paulo-S. Paulo, 1930 - 1 vol.-Doação.

Mario de Assis Moura-Escrituras de compromisso de venda e compra de imoveis-S. Paulo, 1930-1 vol.Dioação.

Mario de Assis Moura-Da intervenção de terceiros (Lei Civil e Processo)São Plaulo, 1932-1 vol.-Doação.

Mario de Assis Moura-Inventarios e partilhas-S. Paulo, 1930-1 vol.Doação.

Mario de Assis Moura-Codigo do Processo Civil e Comercial do Estado de S. Paulo-S. Paulo, 1931--5 vols.Doação.

Mario de Assis Moura-Do pagamento com sub-rogação-S. Paulo, 1933-1 vol.-Doação.

Mario de Assis Moura-Reforma ida Instituição do Juri no Estado de S. Paulo-S. Paulo, 1930-1 vol-Doação.

Mario Braha Henriques-Das socieda- des mercantis irregulares-Belem, 1932 -1 vol.-Doação.

Mario G. D'Oliveira e Cantinho FilhoNulidade fundamental da escritura lavrada pelo escrevente e que o tabelião deixou de assinar-S. Paulo, 1933-1 vol.-Doação.

Mario Pinto Serva-Problemas da constituinte-S. Paulo, 1933-1 vol-Doução.

Mario Pinto Serva-O voto secreto ou a onganização de partidos nacionais -S, Paulo, s/d-1 vol.-Doação.

Mario Pinto Serva-Problemas brasileiros-S. Paulo, 1929-1 vol.-Doação.

Marques Schmidt-A lei da moratoria e o "pactum reservati dominii"-S. Paulo, 1933-1 vol.-Doação.

Marx, Karl-Le Capital-Paris, s/d1 vol.-Doação.

Medeiros e Albuquerque - Parlamentarismo e presidencialismo no Brasil Rio de Janeiro, 1932-1 vol.-Doação.

Mehnert, Klaus-La jeunesse en Russi Soviétique-Paris, 1933-1 vol.-Doação.

Mendes Corrêa-Os criminosos portuguêses-Estudo de antropologia criminal-Coimbra, $1914-1$ vol.-Compra.

Michael, Jerome-Crime, Law and Social Science-London, 1933-1 vol.Doação.

Miranda Neto-Educação e ensino-S. Paulo, 1933-1 vol.-Doação.

Mirkine-Gutzevitch, B-As novas tendencias do Direito ConstitucionalSão Piaulo, 1933-1 vol.-Doação.

Montalbano, G.-Il fondamento dell'imputabilitá-Torinb, $1933-1$ vol.Compra.

Mori, Arturo-Cronica de las Cortes Constituyentes $\longrightarrow$ Madrid, 1933-2 vols -Compra.

Narciso Beriese-Problema fundamental do Brasil-P. Alegre, 1933-1 vol. -Doação.

Niestor Duarte-O direito, noção e norma-Baía, Brasil, 1933-1 vol -Doação.

Nicolau Pero-Nullum crimen in casu -Itapolis, 1933-1 vol.-Doação.

Nitti, F.-Problemas contemporaneos -S. Plaulo, 1933-1 vol.-Doação.

Noé Azevedo-Os interditos possesso- 
rios e as servidões dos concessionarios de serviço publico-S Paulo, 1933 -1 vol.-Doação.

o. Paranaguá-Poditique commerciale internationale-Geneve, $1930-1$ vol. -Doação.

O. Pupo Nogueira-Em torno da tarifa aduaneira-S. Paulo, 1931-1. vol.Doação.

Otaviano Vieira-Casos forenses-S. Carlos, 1909-1 vol.-Doação.

Otaviano Vieira-Os menores perante o Codigo Penal-S. Carlos, 1906-1 vol. -Doação.

Oliveira Ribeiro Neto-Corrigir e prevenir-S. Paulo, 1933-1 vol.-Doação.

Opocensky, Jean-La fin de l'Autriche et la genèse de l'Etat Tchecoslovaque-1928-1 vol.-Doação.

Orestes Guimarães-Sugestões-Florianopolis, 1930—1 vol.-Doação.

Orlando Ferreira-A ilusão capitalista - Uberaba-Minas Gerais, 1933-1 vol. -Doação.

Orlando Ferreira-Capitalismo e comunismo-S. Paulo, 1932-1 vol.Doação.

P. Balmaceda Cardoso-Politica-S. Paulo, 1928-1 vol.-Doação.

P. Baimaceda Cardoso-Estados Unidos da America do Norte-S. Paulo, 1930-1 vol.-Doação.

Pascual, Luis Del Valle-Derecho Politico-Zaragosa, 1932-1934-2 vols.Compra.

Paulo Barreto-Exercicio do comercio por leiloeiros-Sua prova para efeitos fiscais-S. Paulo, 1932-1vol.Doaçãd.

Pedro Montesanti-Errantes da Patria -S. 'Paulo, 1932-1 vol.-Doação.

Pedro Vergara-Flores da Cunba na opinião dos seus contemporaneosP. Alegre, s/d-1 vol.-Doaçã.o.

Penso, Girolamo-Il delinquente instintivo nel progetto Rocco di Codice Penale-Torino, 1929-1 vol-Compra.

Picarolo, A.-O socialismo no BrasilS. Paulo, s/d-1 vol.-Doação.

Picarolo, A-Cristianismo e Democrazia-S. Paulo, 1928-1 vol.-Doação.

Plan Regulador Economico Argentino - Criris mundial-Crisis Argentina-
Monéda-La Plata, 1933-1 vol.-Permuta.

Plano de organização de uma estatistica retrospetiva dos funcionarios (1889-1932)-Rio de Janeiro, 1933-1 vol.-Doação.

Plinio Barreto-Salarios de depositario -S. Paulo, 1933-1 vol.-Doação.

Plinio Barreto-Delegado de carreira -Ação ordinaria de indenizaçãoS. Paulo, 1929-1 vol.-Doação.

Plinio Barreto- 0 convento de S. Francisco e a Faculdade de Direito de S. Paulo-Embargos-S. Paulo, 19331 vol.-Doação.

Plinio Barreto-Deposito, praça e arrematação-S. Paulo, 1931-1 vol.Doação.

Plinio Barreto-Modelo de maridoAção de desquite-S. Paulo, 19271 vol.-Doação.

Plinio Barreto-O Estado de Sitio e a liberdade de imprensa-Ação de indenização-S. Paulo, s/d-1 vol.Doação.

Plinio Barreto-A responsabilidade das emprezas electricas-S. Paulo, 19301 vol.-Doação.

Plinio Barreto-Rescisoria mascarada -S. Paulo, 1928-1 vol.-Doação.

Plinio Barreto-Apelação Civil n. 17.283 -Capital-S. Paulo, 1929-1 vol.Doação.

Plinio Barreto-Anjúrias impressasRazões-S. Paulo, 1924-1 vol.-Doação.

Plinio Barreto-Liquidação de sentença -S. Paulo, 1927-1 vol.-Doação.

Plinio Barreto-O casamento e as molestias contagiosas-S. Paulo, 19281 vol.-Doação.

Plinio Barreto-Uma temeraria aventura forensa-S. Paulo, 1933-1 vol. -Doação.

Plinio Barreto-Um paranoico de grande tomo-S. Paulo, 1932-1 vol.Doação.

Plinio Barreto-Questões criminaisS. Paulo, 1922-1 vol.-D'oação.

Pokrovski, M.-La Revolucion RusaHistoria de sus causas economicas -Madrid, 1931-1 vol.-Compra.

Pontes de Miranda-Introdução á politica cientifica ou fundamentos da 
ciencias positiva do direita-Rio de Janeiro, 1924-1 vol.-Doação.

Projeto de Codigo do Processo Civil e Comercial do Estad o do CearáCeará, 1920-1 vol.-Doação.

Projet.o de Constituição Fed/eral-De acôrdo com as emendas da Bancada Paulista-1 vol.-Doação.

Promessas-"Paz" e a dura realidade -O que quer a Nação Allemã $\rightarrow$ Hamburgo, s/d-1 fol.-Dotação.

Ramiro Berbert de Castro-A edução popular-0 elogio do 2 de julhoRio de Janeiro, 1930-1 vol.-Doação.

Raymond, Allen-Que es la tecnocracia?-Madrid, s/d-1 vol.-Compra.

Recenseamento do Brasil Realizado em 1920-Rio de Janeiro, 1927-1 vol.Doação.

Recenceamento de 1920 -Sessão solene -Rio de Janeiro, 1921—1 vol.-Doação.

Reinack, Adolfo - Los fundamentos aprioristicos del Derecho Civil-Barcelona, 1934-1 vol.-Compra.

Reissener, Larissa-Homens e maquinas-miserias e capitalismo-S. Paulo, 1932-1 vol.-Doação.

Reparaz, Gonzalo de-Geografia y politica-Barcelona, 1929-1 vol.-Doação.

Resumo da Estatistica do Ensino Primario Geral no Brasil em 1931Rio de Janeiro, 1932-1 vol.-Doação.

Resumo da Estatistica Geral do Ensino no Brasil em 1931-Rio de Janeiro, 1933-1 vol-Doação.

Rezzara, Guido-Della apertura di credito in conto corrente-Torino, 1926 -1 vol.-Compra.

Rodrigo Otavio-Dicionario de Dineito Internacional Privado-Rio de Janeiro, 1933-1 vol.—Doação.

Russell, Bertrand-El socialismo, el anarquismo y el sindicalismo-Madrid, 1932-1 vol.-Doação.

Rui Barbosa-Comentarios á Conrtituição Federal Brasileira—S. Paulo, 1933 2 vols.-Compra.

Schupfer, Francisco-Il diritto delle obligazioni in Italia nell'Etá del ri-
sorgimento-Torino, 1921-3 vols.Compra.

Seillière, Ernest-Rcmantisme et démocraitie-Paris, 1932-1 nol.-Doação.

Siegfried, André-Tableau des Partis em France-Paris, s/d-1 vol.-Doação.

Smith College Studies in Social Work -Northampton, Mass., 1930-1 vol.Doação.

Sinopse do recenseamento realizado em 1 de setembro de 1920—Rio de Janeiro, 1926-1 vol.-Doação.

Slanviski, Stanislas - L'accès de la Pologne à la Mier et les interêts de la Prusse Orientale-Paris, 1925-1 vol. -Doação.

Spirito, Ugo-I fondamenti della Economia Corporativa-Milano, Roma1 vol.-Permuta.

Statistiques des Migrations-(Les)Genove, 1932-1 vol.-Compra.

Sud Menucci-Brasil desunido-S. Paulo, 1932-1 vol.-Daação.

T. de Sousa Lobo-S. Paulo na Federação-S. Paulo, 1923-1 vol.-Doação.

T. de Sousa Lobo-Brasil Confederado -S. Paulo, 1933-1 vol.-Doação.

Teodomiro Dias-Tio contra pai-S. Paulo-1 1929-1 vol.-Doação.

Teodomiro Dias-O Banco Evolucionista e o Regente Feijó-S. Paulo, 1929-1 vol.-Doação.

Teodomiro Dias-Liquidação de danos oriundos de incendio-S. Paulo, 1934 -1 vol.-Doação.

Teodomiro Dias-Embargos. Obras • manobras do Banco Evolucionista -S. Paulo, 1929-1 vol.-Doação.

Teodomiro Dias-Do suprimento de energia eletrica-S. Paulo, 1933-1 vol.-Doração.

Teodomiro Dias-Sentença que preconiza o estelionato-S. Paulo, 19321 vol.-Doação.

Teofilo Dias de Mesquita-Teses e dissertação apresentadas á Faculdade de Direito de S. Paulo-S. Paulo, 1883-1 vol.-Doação.

Tomaz Lessa-Um esbulho judicialMiemorial-S. Paulo, 1930-1 vol.Doação.

Tomaz Lessa-Da cessão de crédito hi- 
potecario-S. Paulo, 1930-1 vol.Doação.

Tomaz Lessa-Apelação oivil n. 8913 de Espirito Santo do Pinhal-RazõesS. Paulo, 1927-1 vol.-Doação.

Tomaz Lessa-Da integralização do imovel vendido com divisas certas -S. Paulo, 1930-1 vol.-Dọação.

Tomaz Lessa-Da participação nos lucros sociais e do direito do exame integral de livros--S. Paulo, 19331 vol.-Doação.

Tomaz Lessa-Um caso de desapropriação sem utilidade pública-S. Paulo, 1932-1 v.ol.-Doação.

Tomaz Lessa-Teodolindo Castigfione -Da clausula "rebus sic stantibus" nois contratos de compra e venda -S. Paulo, 1933-1 vol_-Doação.

Tomaz Pimentel e Ticiano PimentelCodigo do Processo Civil e Comercial do Estado de S. Paulo, 1933-1 vol.-Doaçãn.

Tostes Malta-Do flagrante delito-S. Paulo, 1933-1 vol.-Compra.

Tristão de Athayde-O problema da burguezia-Rio de Janeiro, 1932-1 vol.-Doação.

Ulisses Doria, Anibal Quintella Junior e Paulo Barrero-Recurso crimePrivilegio de invenção-S. Paulo, s/d-1 vol.-Doação.

Urstin, Mauricio-Criminalidad e Psicose-B. Aires, 1930-1 vol.-Doação.

Valois, Greorges-Un nouvel âgè de l'humanité-Paris, 19:29-1 vol. Doação.

Vandervelder, Emile-Etudes Marxistes-Bruxelles, 1930-1 vol.-Doação.

Vervaeck, L.-Le applicazioni pratiche della antropologia criminale - Torino, 1925-1 vol.-Compra.

Vianex, Georges-La révolution ou la guerre-Parir, s/d-1 vol.-Doação.

Vicente Piragibe-Legislação penal do Brasil e do Estrangeiro-S. Paulo, 1932-2 vols.-Compra.

Vicente Ráo-Democracia e a eção social do Estado-S. Paulo, 19311 vol.-Dicação.

Vitor Viana-O regime fascista e a democracia-A utpia reacionaria e as realidades brasileiras-Rio de Janeiro, 1933-1 vol.-Compra.

Vitor Viana-A constituição dos Esta- dos Unidos-Rio de Janeiro, 1933vol.-Compra.

Vieira Ferreira-Ementas e emendas ao projeto de Codigo Civil-Rio de Janeiro, 1912-1 vol.-Doação.

Villanuova y Morales e Ricardo Royo -O direito de morrer rem dorS. Paulo, 1933-1 vol.-Doação.

Virgilii, Filippo-Il problema della por polazione-Milano, $1924-1$ vol.Compra.

Waldemar Ferreira-A responsabilidade por acidente no transporte gracioso por automovel-S. Paulo, 1930 -1 vol.-Dcação.

Waldemar Ferreira-Do regime de bens do estrangeiro - S. Paulo, 1922-1 vol.-Doração.

Waldemar Ferreira-Ação de reivindicação das terras do Ribeirão Doce e Batalha-S. Paulo, 1926-1 vol.Doação.

Waldemar Ferreira-O requerimento doloso de falencia e a obrigação de indenizar-S. Paulo, 1931-1 volDoação.

Waldemar Ferreira-A caução de co nhecimentos por comissarios de café -S. Paulo, s/d-1 vol.-Doaçāo.

Waldemar Ferreira-Questões de direito comercial-2." serie-S. Paulo 1932-1 vol.-Doação.

Waldemar Ferreira-As diretrizes do direito mercantil brasileiro- Lisboa, 1933-1 vol.-Doação.

Waldemar Ferreira-A suspensão dos embarques de café e os contratos de compra e venda-S. Paulo, 1931-1 vol.--Doação.

Waldemar Ferreira-A conferência de bens do socio e a sociedade leonina -S. Paulo, 1930-1 vol.-Doação.

Waldemar Ferreira-As sesmarias e terras devolutas-S. Paulo, 1931- 1 vol-Doação.

Waldemar Ferreira - A propósito de remuneração de mandatáriós - $S$. Paulo, 1929-1 vol.-Doação.

Waldemar Ferreira-O endosso pignoraticio de conhecimentos ferroviarios -S. 'Paulo, 1931-1 vol.-Doação.

Waldemar Ferreira e Eliezer A. de Toledo-Um cado de procuração "em causa propria"-S. Paulo, 1931-1 vol.-Doação. 
Waldemar Ferreira e Targino Ribeiro -Apelação civil n. 5.593-Rio de Janeira $s / d-1$ vol.-Doação.

Waldemar Ferreira e Tomaz Lessa-A proposito de um cheque-S. Paulo, 1931,--1 vol.-Doação.

Waldemar Ferreira e Tomaz Lessa Em prol de uma instituição de caridade-S. Paulo, $1924-1$ vol.Doação.

Waldemar Ferreira e Tomaz Lessa A responsabilidarde do socio comanditario-São Paulo, 1933-1 vol.Doação.

Whitaker-Júri-Estado de S. Paulo1930, 6.a ed:-Doação-1 vol.

Zanobinii, Guido-Le Sanzione Amministrative-Torino, $1924-1$ vol.Compra.

\section{FILOLOGIA E LINGUISTICA - 4}

Larousse, $\mathrm{P}$-Grammaire supérieureParis, s/d-1 vol.-Doação.

Grammaire de l'Académie françaiseParis, 1933-1 vol.-Doação.

Plinio Ayrosa-Primeiras noções de tupí-São 'Paulo, 1933-1 vol.Doação.

\section{CIENCLAS PURAS -5}

Matematicas, fisica, quimica, biologia, antropologia, etc.

Berardinelli, W-Biatipollogia criminal Rio de Janeiro, $1933,-1$ vol.Compra.

Brunelli, Gustavo-Le teorie sul'origine e l'evoluzinoe della vita-Bologna, 1933-1 vol.-Compra.

Darwin, Leonard-Que és la eugenesia?-Madrid, 19:30-1 viol.-Compra.

Eisenmenger,G-La physique, son rôle et ses phénomènes dans la vie quotidienne.-Paris, 1921-1 volDoação.

J. Barbosa Rodrigues-Les noces des palmiers-Bruxelle's, 1909-1 vol.Permuta.

J. Barbosa Rodrigues-Sertum Palmarum Brasiliensium ou relation des palmiers n'ouveaux du BrésilBruxelles, 1903—2 vols.-Permuta.
J. Rodriguesi Mereje_Apontamentos de quimica toxicologica-São Paulo, 1922 -1 vol.-Doação.

Kirchberger, Paul-La théorie de la relativité exposée s'ans mathématiques-Paris, 1922-1 vol.-Doação.

Kleiben-Kleister-Tratado popular de física-Barcelona, 1919-1 vol.Doação.

Moch, Gaston-Initiation aux théories d'Einstein-Paris, s/d-1 vol-Doação.

Ortiz, C. Silva Figuera y Stuardo, CManipulaciones de zoologia-Chile, 1933-1 vol.-Doação.

Otavio Domingues-Eugenia-seus propositos, suas bases, seus meiosSão Paulo, 1933-1 vol.-Doação.

Renato Kehl-Politica eugênica-Porto, 1933-1 vol.-Doação.

Renato Kehl-A utopia da felicidade coletiva-Porto, 1933-1 vol.-Doação. Roquette Pinto-Ensaios de antropologia brasiliana--São Paulo, 1933-1 vol-Compra.

\section{CIênClas APLICADAS - 6}

Medicina, Engenharia, Comércio, etc.

A. C. Pacheco e Silva-Cuidados aos psicopatas-São Paulo, 1930- 1 vol. -Doação.

Binet, Jéon-Questions physiologiques d'actualité-Paris, 1927--1 vol.Doação.

Cerda, 'Pedro Aguirre-El problema in. dustrial-Chile, 1933-1 vol.-Doação.

Eugenio George-Soros e vacinas-Rio de Janeiro, 1934-1 vol.-Doação.

Francisco d'Auria-Contabilidade pública-São Paulo, 1927-1 vol.-Compra.

Germano P. Franck-A Amazonia e o futuro de sua industria florestalCeará 1932-1 vol.-Doação.

Henrique Dumont 'Villares-Ligeinas notas sôhre economia agricola-São Paulo, 1931-1 vol.-Doação.

José Benedito de Moraes Leme-O problema venéreo-São Paulo, 1926-1 vol.-Doação.

Raul Ribeiro da Silva-O problema da siderurgia no Brasil-Rio de Janeiro, 1922-1 vol.-Doação.

Reinaldo Ribeiro da Silva-Anatomia 
e figiologia humanas-São Paulo, 1931 -1 vol.-Doação.

Sebastião Medeiros-Problemas do Café -São Paulo, 1930-1 vol.-Doação.

\section{BELAS ARTES - 7}

Arte, jogos, divertimentos.

J. Canuto Mendes de Almeida-Cinema contra cinema-São Paulo, 1931-1 vol.-Doação.

Massarini, Tullo-L'art à Paris, Paris, '1880-1 vol.-Doação.

Ricardo Arruda-A arte de roubar no jogo-São Paulo, 1926-1 vol. Doação.

Rios, H. Ginez de los-Teoria de la literatura $y$ de las artes-Buenos Aires.

\section{LITERATURA -8}

Adelaide de Castro Alves GuimarãesO Imortal-Rio de Janeiro, 1934-1 vol.-Doação.

Albino Forjaz de Sampaio-Antonio Nobre-Losboa, 1919-1 vol.-Doação.

Alcides Maya-Machado de Assis-Rio de Janeiro 1912-1 vol.-Doação.

Alfredo Ellis-Discursos parlamentares-São Paulo, 1914-3 vols.-Doação.

Alegretti Filho-Ouro velho-São Paulo, 1933, 1 vol.-Doação.

Almeida Garrett-Obras completasRio de Janeiro, 1854, 2 vols.-Permuta.

Altino Arantes-Disse... São Paulo, 1923, v vol.-Dơação.

Altino Aranties-Bonum Opus-São Paulo, 1930-1 vol.-Doação.

Aluizlo de Azevedo-O Cortiço-Rio de Janeiro, s/d-1 vol. -Daação.

Alvares de Azevedo-Obras completas -Rio de Janeiro, s/d-3 vols.Permuta.

Amadeu Nogueira-Misérias-São Paulo, 1932, 1 vol.-Doação.

Andreiev, L.-Dies Irae-Madrid, 1920 -1 vol.-Duação.

Angelo Guarinello-A salada da vida -Coritiba, 1930, 1 vol-Doação.

Antonio Sergio-Nota sôbre os sonetos e as tendencias gerais da filosofia de Antero de Quental-Lisboa, 1909. 1 vol.-Doação.

Antonio Torres e Adoasto de GodoyDa correspondencia de João Episco po-Rio de Janeiro, 1917, 1 vol.--Doação.

Aquilino Ribeiro-Anatole FranceRio' de Janeiro, 1923, 1 vol-- Doação.

Aragon, Louis-Traité du style-Paris, 1928, 1 vol.-Doação.

Arnoux, Jacques-Palavras de um redivivo-São Pau'lo, 1928, 1 vol. Doação.

Arquivos del General Miranda-Caracas, 1929, 2 vols.-Doação.

Armando Caiuby-O mistério do Cabaré-São Paulo, 1931-1 vol.-Doação.

Augusto de Lacerda-As duas pátrias -Porto, 1908, 1 vol.-Dơação.

Barrie, J. M.-Lady Nicotine-New York, 's/d-1vol.-Doação.

Belmonte-Assim falou Juca Pato-São Paulo, 1933, 1 vol.-Doação.

Benedito Salgado-Microcosmo-São Paulo, 1924, 1 vol.-Doiação.

Benjamin Constant-Guria-São Paulo, a/d-1 vol.-Doação.

Brazilio Machado-Discurso-São Paulo lo, 1887, 1 vol.-Doação.

Brazilio Machado-Dias de ImprensaSãio Paulo, 1900-1 vol.-Doação.

Brazilio Machado-Madressilvas-Porto, 1876, 1 vol.-Doação.

Brazilio Machado-Discurso proferido no dia 8 de dezembro por ocasião da colação de grau aos bacharelandos de 1894—São Paulo, 1894, 1 vol.— Doação.

Campodarbe, D. Demetrio Espurz YDiscurso leido en la solemne Apertura del Curso LAcademico de 193334. Oviedo-1934, Um Folheto, Doação.

Canto e Mello-Mana Silvéria-São Paulo, 1914, 1 vol.-Doação.

Canto e Mello-Bucolica-São Paulo, 1923, 1 vol.-Doação.

Canto e Mello-Reliquias da memória -São Paulo, 1920-1 vol.-Doação.

Canto e Mello-Alma em delirio-São Paulo, 1914, 1 vol-Doação.

Carlos Sampaio-Conferencijas-Ribeirão Preto, 1 vol-Doação. 
Carrel, Arnaud-Oeuvres politiques et littéraires-Paris, 1857, 5 vols.-Doação.

Cartas de Machado de Assis a Euclides da Cunha-Rio de Janeiro, 1931, 1 vol.-Doação.

Castilho-Obras completas-Do 2. ao 80. vol.—Lisboa, 1903-1910—23 vols. -Permuta.

Castro Alves-Aideus meu canto-Baía, 1894, 1 follh.-Doação.

Celestino Silveira-Carne moça e outras banalidades-Rio de Janeiro, 1931, 1 vol.-Doação.

Cendrars, Blaise-Les confessions de de Dan Yack-Paris, 1929, 1 vol.Doação.

Chiado-Obras completas-Lisboa, 1889, 1 vol.-Permuta.

Congresso Artistico e Teatral realizado no Rio de Janeiro, por iniciativa da Sociedade Brasileira de Autores Teatraes-São :Paulo, 1924, 1 folh.-Doação.

Cunha Bueno Junior-A purificação de um heptalogo-S. Paulo, 1934, 1 vol. -Dciação.

Damasceno Vieira-A Castro AlvesBaia-1898, 1 vol.-Doação.

Delioz, Paulo-Rémy de Gourmont et son ceuvre-Paris, 1909, 1 vol.Doação.

Delteil, Joseph-Le mal de coeur-Paris $s / d-1$ vo.l.-Doaçãio.

D'ors, Eugenio-El nuevo. GlosarioMadrid, 1921, 1 vol.-Doação.

D'ors, Eugenio-Glosario-Madrid, 1920, 1 vol.-Doação.

Duisserg, Karl-Vortrage und reden aus den Jahren 1922-1933,-Berlim, 1933, 1 vol.-Doação.

E. de Castro Rebel'lo-Discurso-Rio de Janeirob 1934, 1 vol.-Doação.

Ȩa de Queiroz-Revista de Portugal $\longrightarrow$ Porto, 1899, 4 vols.-Permuta.

Eça de Queiroz-Uma campanha alegre, Das Farpas-Lisboa, 1890, 1 vol. -Permuta.

Emilio de Menezes-Poresias-Rio de Janeiro, 1909-1 vol.-Doação.

Emilio de Moura-Ingenuidiade-Belo Horizonte, 1931, 1 vol-Doação.

Epitacio Pessoa-Discurso pronunciado por ocasião da visita a São Paulo, em
Agosto de 1921-São Paulo, 1922-1 folh.-Doação.

Eugenio de Castro-Constança-Coimbra, 1900, 1 vol.-Doação.

Eurico de Goes-Horas de lazer-Rio de Janeiro, 1914, 1 vol.-Doação.

F. de Aquino Corrêa-Castro Alves e as moças-Rio de Janeiro, 1933, 1 vol.-Doação.

Fernando da Cunha Filho-NinféasSã.o Paulo, 1876, 1 vol.-Doação.

Fernando Rodrigues-Férias em terras distantes-Rio de Janeiro, 1933, 1 vol.-Doação.

Floretti, I-Il cantico delle creatureMilano, 1920, 1 vol.-Doação.

Francisco Brandt-Em férias-São Paulo, 1889, 1 vol.-Doação.

Geraldy, Paul-Eu e você (Trad. de Guilherme de Almeida-São Paulo, 1932, 1 vol-Doaçãio.

Gofnedo da Silva Telles-A fada nuaSãlo Paulo, 19:23, 1 voil.-Doação.

Gofredo dia Silva Telles-O martir da noite-Rio de Janeiro, 1915, 1 vol.Doação.

Gomes Cardim-O jequitibá-São Paulo, 1930, 1 vol.-Doação.

Gomes Cardim-Discurso-São Paulo, 1929, 1 vol.-Doação.

Gomes Cardim-Não dá passarinhoSoã ,Paulo, 1928, 1 vol.-Dıação.

Gomes Cardim-Caboclos-São Paulo, 1930, 1 vol.-Doação.

Gomes Cardim-Simplicidade-São Paulo, 1929, 1 vol-Doação.

Gomes Cardim-Lélé-São Paulo, 1929, 1 vol.-Doação.

Gomes Cardim-A esposa de S. Exa.São Paulo, 1928, 1 vol.-Dóação.

Gomes Cardlim-A procuração-São Paulo, 1915, 1 vol.-Doação.

Gomes Cardim-O cornet-São Paulo, 1915-1 vol.-Doação.

Gomes Cardim-Lever de Rideau e monologos-São Paulo, 1908, 1 vol.Doaação.

Gomes Cardim-Um grande momento $\longrightarrow$ São Paulo, 1910, 1 vol.-Doação.

Gomes Cardim-Chi lh'a dettoi?-São Paulo, 1909, 1 vol.-Doação.

Gomes Cardim-Uma prova de consideração-São Paulo, 1929, 1 vol.Doação. 
Gomes Cardim-Monologos e sainetes -São Paulo, 1929, 1 vol.-Doação.

Govoni, G.-L'inaugurazione della primavera-Milano $s, d-1$ vol.-Doação.

Guerra Junqueiro-A musa em férias -Lisboa, 1913, 1 vol.-Doação.

Guilherme de Almeida-A dança das horas,-São Paulo, 1928, 1 vol.-Doação.

Guilherme de Almeida-Nós—São Paulo, 1930—1 vool.-Doação.

Guilherme de Almeida-SimplicidadeSão Paulo, 1929, 1 vol.-Doação.

Guilherme de Almeida-O Gitanjali de Rabindranath Tagore-São Paulo, 1932, 1 vol.-Doação.

Guilherme de Almeida-Você-São Paulo, 1933, 1 vol.-Doação.

Guilherme de Almeida-Livro de horas de Soror Dolorosa-São Paulo, 1928, 1 vol.-Doação.

Guilherme de Almeida-Carta a minha noiva-São Paulo, 1932, 1 vol.-Doação.

Heine, Henri-Pages Choisies-Paris, 1912, 1 vol.-Doação.

Herreros, Pedro-Buenos Aines grotesao $\mathbf{y}$ otros motivos-Buenos Aires 1922, 1 vol.-Doação.

Istrati, Panait-La Russie nue-Paris, 1929-1 vol.-Doação.

Istrati, Panait-La Russie nue-Paris, 1 vol.-Doação.

J. C. Ataliba Nogueira-Santo Antonio na tradição brasileira-São Paulo, 1933, 1 vol.-Doação.

J. Pinto Antunes-Alvares de Azeverdo -São Paulo, 1932, 1 vol.-Doação.

Jacy de Assis-Saudade-São Paulo, 1932, 1 vol.-Doação.

João Alfredo Botelho de Miranda-Um conto e 40 poemas-São Paulo, 1931, v vol.—Doação.

João Mendes de Almeida Junior-Alocução-São Paulo, 1893, 1 vol.-Doaçã̀o.

João do Rio-A alma encantadora das ruas-Rio de aneiro, 1910, 1 vol.-Doação.

Jorge de Lima-Poemas-Rio de Janeiro, 1932, 1 vol.-Doação.

José Bonifacio Ferreira-Maria Angelica-São Paulo, 1933, 1 vol.-Doação.
Kisch, Egon Erwin-O Paraiso Norte Americano-São Paulo, 1933, 1 vol.Doação.

Lamartine $F$. Mendes- Aguas passadas -São Paulo, 1932, 1 vol-Doação.

La Serna, Ramon Gomez de-Variaciones-Madrid, s/d-1 vol.-Doação.

Luiz Candido Furtado Coelho-A memória do grande João Caetano dos Santos-Rio de Janeiro, 1884, 1 vol.Doação.

Manuel Galvez-Jornadas de agoniaBaía, 1931, 1 vol.-Doação.

Mario Carli-A canção do sanguePorto Alegre, 1932, 1 vol.-Doação.

Mario Sette-A mulher de meu amigo São Paulo, 1933, 1 vol.-Doação.

Marta de Holanda-Delirio do nadaRecife, 1930, 1 vol.-Doação.

Matthews, T. I.-A caminho da forca Rio de Janeiro, 1933, 1 vol,-Doação.

Menna Barreto de Barros Falcão-No fogo..., Recife, 1922, 1 vol-Doação.

Miguel Rizzo-O varão de dores-São Paulo, 1931, 1 vol.-Doaçãó.

Montaigne-Les essais-Londres, 1919, 6 vols.-Permuta.

Motta Filho-Introdução ao estudo do pensamento nacional-São Paulo, 1926, 1 vol.-Doação.

Mugnier, Henri-La couronne à la France-Paris, 1918, 1 vol.-Doação.

Newton Belleza-A mulher que virou homem-Porto Alegre, 1932, 1 vol.Doação.

Nobrega da Siqueira-Faz de contaSão Paulo, 1933, 1 vol. vol.-Doação.

Nordau, Max-Vus du dehors $\longrightarrow$ Paris, 1903, 1 vol.-Doação.

Nova Cruzada a Castro Alves-Baía, 1908-1 vol.-Doação.

Odecio Camargo-Insônia-Rio de Janeiro, 1931-1 vol.-Doação.

Olegario Marianno-Ultimas cigarrasRio de Janeiro, 1915, 1 vol.-Doação.

Povidio M. Chaves-CancioneiroPorto Alegre, 1933, 1 vol.-Doação.

Paulo Eiró-Sangue limpo-São Paulo, 1963, 1 vol.-Doação.

Paulo Setubal-A Marquesa de Santos-São Paulo, 19331 yol.-Doação.

Paulo Setubal-A's maluquices do Imperador-São Paulo, 1932, 1 vol.Doação. 
Paulo Setubal-Alma cabocla-São Paulo, 1929, 1 vol.-Doação.

Paulo Setubal-OS Irmãos Leme-São Paulo, 1933, 1 vol.-Doação.

Paulo Setubal-Nos bastidores da Historia $\longrightarrow$ São Paulo, 1930, 1 vol.Doação.

Paulo Setubal-O Principe de NassauSão Paulo, 1933, 1 vol.—Doação.

Paulo Setubal- A Bandeira de Fernão Dias-São Paulo, 1928, 1 vol.Doação.

Paz, Henrique-Abelardo Lobo-Cordoba, 1933, 1 vol-Doação.

Pedro Luiz Pereira de Souza-Terribilis Dea-Rio de Janeiro, 1933, 1 vol. -Doação.

Percival de Oliveira-Portugal de hoje e de amanhã-São Paulo, 1933, 1 vol. -Doação.

Pirandello, Luigi-Vestire gli inudiFirenze, s/d-1 vol.-Doação.

Pirandello, Luigi-La Signora Mardi una e due-Firenze, 1922, 1 vol.Doação.

Pirandello, Luigi-L'Uomo, la Bestia e la Virtú-Firenze, 1922, 1 vol.Doação.

Pirandellob Luigi-Enrico IV-Firenze, 1922, 1 vol.-Doação.

Pirandello, Luigi-Come prima meglio di prima-Firenze, 1921, 1 vol.Doação.

Plinio Barreto-Paulo Mazoldi-São Paulo, 1922, 1 vol.-Doação.

Ramalho Ortigão-As Farpas-Lisboa, 1887, 12 vols.-Permuta.

Raul Brandão-Humus-Lisboa, s/d.1 vol_-Doação.

Renato Kehl-Discurso_Rio de Janeiro, 1933, 1 vol.-Doação.

Ricardo Gonçalves-Ipês-São Paulo, 1922, 1 vol.-Doação.

Ronaldo de Carvalho-Poiemas e Sonetos-Rio de Janeiro, 1919, 1 vol.Dolação.

S. Flores de Abreu-Na terra das palmeiras-Rio de Janeiro, 1931, 1 vol. -Doação.

Salvador José Ferretra Coelho-Passeio a minha terra-S. Paulo, 1860, 1 vol.-Doação.

Sampaio Freire-Versos de outro tempo-S. Paulo, 1926, 1 vol.-Doação.
Sampaio Freire-Ensaios-Campinas, 1915, 1 vol.-Doação.

Schwob, Marcel- Le livre de Monelle-Paris, 1923, 1 vol.-Doação.

Sem-Benelli-L'amione dei tre ReMilano s/d.-1 vol.-Doação.

Sergio de Chessin-A noite que vem do Oriente-Porto Alegre, 1932, 1 vol.-Doação.

Shakspeare-Otélo-Porto, 1911, 1 vol. Permuta.

Shakspeare-O Rei Lear-Porto, 1911, 1 vol.-Permuta.

Shakspeare-Macbeth-Madrid, 1919, 1 vol.-Doação.

Silva d'Azevedo-O Exercito desconhecido-São Paulo, 1933, 1 vol.-Doação.

Silvio de Almeida-Estudos Camonianos-São Paulo, 19.25, 1 vol.- Dpiação.

Siqueira Junior-Haikas-Rio de Janeiro, s/d.-1 vol.-Doação.

Sudermann, H-Magda ou volta á casa paterna-São Paulo, 1923, 1 vol.Doação.

Tapajós Gomes-Lua de Mel-Rio de Janeiro, 1916, 1 vol.-Doação.

Théoi-Filho - Impressões transatlânticas-Rio de Janeiro, 1931, 1 vol.Doação.

Torre, Guillermo de-Literaturas europeas de vanguardia-Madrid, 1925, 1 vol.-Doaçãio.

Veiga Miranda-Maria Cecilia e outras historias-Rio ide Janeiro, 1930, 1 vol.-Doação.

Vigny, Alfred de-Oenuvre poétiqueParis, 1920, 1 vol.-Doação.

Vargas-Vila-Diel rosial pensanteMexico, 1914, 1 vol.-Doação.

Werth, Léon-Voyages avec ma pipe -Paris, 1920, 1 vol.-Doação.

Zuccante, Giuseppe-Figure e dottrine nell'opera di Dante-Milano, 19211 vol.—Doação.

\section{HISTORLA - 9}

Historia, Geografia politica, viagens, biografia, Heraldica, etc.

A. Pompeo-Conferências-São Paulo, 1933, 1 vol.—Doação.

Album de Barretos-São Paulo, 1933 1 vol.-Doação. 
Americano do Brasil-Súmula da Historia de Goiás-Goiás, 1932, 1 vol. -Doaçãio.

Americo de Novaes-Conferências

Antonio Luiz dos Santos WerneckO positivismo republicano na Academia-São PPaulo, 1880, 1 vol.Doação.

Antoine Renard-São Paulo é istoSão Paulo, 1933, 1 vol.-Doação.

Araujo Líma-A Amazonia, a terra e o homem-Rio de Janeiro, 1933, 1 voll--Doiação.

Bates, Henry Walter-The natunalist on the River Amazons-New York, 1932-1 vol.-Compra.

Candidoi Motta Filho-Uma Grande vida-Bernardino de Campos-São Paulo, 1931, 1 vol.-Compra.

Clovis Ribeiro-Brazões e Bandeiras São Paulo, 1933, 1 vol-CCompra.

Benda, Julien-Histoirel des Français dans leur effort pour devenir une nation-Paris, 1933, 1 voli.-Doação.

Ciro Costa e Eurico de Groes-S.ob a metralha...-São Paulo, 1924, 1 vol. -Doação.

Djalma Frjaz-O Senador VergueiroSão Paulo, 1924, 1 vol.-Dloação.

Documentos interessantes para a historia de São Paulo-Tomo 47-São Paulo, 1929, 1 vol.-Doação.

Duisberg, Karl-Meine Lebeserinnerungen-I.eipzig-1933, 1 vol.-Doação.

E. de Castro Rebello--Mauá-Rio ide Janeiro, 1932, 1 vol.-Doação.

Eugenio Egas-Estudos-São Paulo, 1932, 1 v.ol.-Doaçãlo.

Eurico de Goes-A Bandeira positivista-São Paulo, 1927, 1 vol.-Doação.

Fedoroff,' Michel-La Russie sous le régime communiste-Paris, 1926, 1 vol.-Doação.

Fernando Nobre-As fronteiras do Sul -São Paulo, 1922, 1 vol.-Doação.

Fleg, Edmond-Moysès-Paris, 1926, 1 vol.-Doação.
J. M. Pereira da Silva-Plutarco Brasileiro-Rio de Janeiro, 1874, 2 vols. - Permuta.

João Mendes de Almeida Junior-Monografia do Municipio da Cidade de São Paulo-São Plaulo, 1882, 1 vol. -Doação.

José Torres de Oliveira-Anchieta e a pacificação dos indilos-São Paulo, 1933-Doação.

Lange, Christian-Russia, The Revolution and the War-Washington, 1917, 1 vol.-Doação.

Le Bon, Gustave-Bases scientifiques d'une philosophie de l'histoireParìs, 1931, 1 vol.-Doação.

Lefevre, Louis Raymond-La vie de Cyrano de Bergerac-Paris, 1927, 1 vol.--Doação.

Joaquim Manoel de Macedo-Ano biografico brasileiro-Rio de Janeiro 1876, 4 vols. $\longrightarrow$ Permuta.

Mehring, Franz-Karl Marx, historia de su vida-Madrid, 1932, 1 vol.Doação.

Nassi, S-Histoire de la Syrue-Paris, 1924, 1 vol.--Doação.

Prévost, Michel-Le divorce pendant la Révolution-iParis, 1908, 1 vol.Doação.

Rodes, Jean-La Chine nationalisteParis, 1931, 1 vol.-Doação.

Saint-Adolphe, J. C. 'R. Milliet deDicionario gieografico, historico e descritivo do Brasil-Paris, 1863, 2 vols. -Permuta.

Spalding, Walter-A' luz da Historia -Porto Alegre, 1933, 1 vol.-Doação.

Tomás Jefferson-O Partido Republicano na Provincia de São PauloRio de Janedro, 1877, 1 vol.-Doaçăb.

Vilhena de Moraes-Caxias-Rio de Janeiro, 1934, 1 vol.-Compra.

Visconde de Taunay-Imperio e $\mathrm{Re}$ publica-Rio de Janeiro s/d-1 vol -Doação.

Warden, M. David.-Histoire de l'Empire du Brésil-Paris, 1832, 2 vols. -Permuta. 